\title{
Phreatische Fauna in Ljubljansko polje (Ljubljana-Ebene, Jugoslavien) - ihre ökologische Verteilung und zoogeographische Beziehungen
}

\author{
B. Sket* und F. Velkovrh*
}

\begin{abstract}
SUMMARY
The phreatic basin of Ljubljansko polje (polje = plain, field) recharges its water supply mainly from the Sava river-bed and at a few other locations where connections with karstic subterranean waters might exist and only up to $15 \%$ from precipitation. An important zone of infiltration in the river-bed is the bottom and not the bank which is to a large extent watertight due to organic debris (rests of Sphaerotilus e.g.). The main water-body moves about $10 \mathrm{~m} / \mathrm{day}$, there are however some local jets with far higher speeds.

Yearly amplitudes of water temperatures are high near the river but in the center of the plain only a couple of centigrades. Oxygen saturation is in the open river-water $100 \%$, dropping to 40 $60 \%$ just $1 \mathrm{~m}$ into the phreatic.

True stygopsammal animals are represented here only by a few species and specimen in spite of the fact, that the interstices in the gravel are mostly filled with finer sediments. Remarkable is also the scarcity of Nematodes and the near absence of Acarina (compare with Danielopol 1976).

Only a few specimen of the river benthos (Chironomidae, Tipulidae, Leuctra supp., Baetis spp.) penetrate the interstitial water (compare with Ruffo 1961, Danielopol 1976) and only Naididae are more frequent there. However, many epigean animals occur in interstitial waters in the periodically flooded gravel-banks; one can explain this with oscillations of the water level. Some epigean animals (creno- and troglophilic) are quite regularly represented in the phreatic near the river, but have not been found in the river-bed.

The distribution of phreatic species within the studied water-body seem to be controlled mainly by the presence of food supplies and the consequent competition among species. The same is true for the speed of the water current and some other factors which are less easily defined. The characteristics of the substratum as well as $\mathrm{O}_{2}$-saturation and other characteristics of the water seem to have little influence on the fauna.

The energetically (food-) rich neighbourhood of the river is inhabited by a number of species in quite dense populations while the central parts of the phreatic water body exhibit a great poverty of species and of specimen. However, some species live here, which don't occur in the presence of larger food supplies - and of greater competition (Niphargus serbicus). The higher current speed seems to prevent settlement of some species (Cyclopoida, Proasellus deminutus) while some are bound to such habitats (Proasellus vulgaris).

Some species exhibit a high degree of euryvalency inside the stygopsephale habitats (Niphargus longidactylus e.g.), while some are highly specialized. Some of them form dense populations (comparatively dense even in energetically poor places) while others exhibit even in most favourable conditions very low densities (Niphargus jovanovici multipennatus).
\end{abstract}

The present fauna is zoogeographically very diverse. Some species are distributed throughout

* Institut za biologijo Univerze und Biotehniska fakulteta, YU 61001 LJUBLJANA, pp. 141, Yugoslavia.

Die Untersuchungen wurden von Raziskovalna skupnost Slovenije (Research Council of Slovenia) finanziell unterstützt. 
Europe; some reach from Central Europe to the borders of Dinaride Karst (Bogidiella albertimagni) and some even penetrate it (Trichodrilus pragensis, Acanthocyclops kieferi). Bogidiella semidenticulata, Niphargus pectinicauda, Hadziella deminuta seem to be limited to the higher reaches of the Sava River. All of the above mentioned animals live regularly in interstitial waters and only sporadically in karstic hypogean waters.

Niphargus stygius is here the only animal of a certainly karstic provenience; inside the plain it is limited to a completely special habitat. It is very likely that the entire Proasellus-deminutusgroup has developed in interstitial waters of larger plains which are in contact with karstic areas; some species penetrated from the plains into the karst rather than the reverse. To the contrary (judging from the distribution of the genera) karstic waters seem to be the cradle of Hauffenia and Hadziella. Such a sharp delimitation between cave- and interstitial fauna resp. in this area is very noteworthy. Both faunas live here in abundance and in close contact. It is very probable that particularly high competition and specialization of both faunas, caused by their richness and diversity, prevent mixing of species.

\section{EINLEITUNG}

Das Ljubljansko polje (polje = Feld, Flur, Ebene!) ist eine junge, tektonische, angefüllte, vorwiegend im Pleistozän entstandene Einsenkung, deren Boden wasserundurchlässige permo-karbonische Schiefer und Sandsteine bilden. Es hat die Form einer Schüssel, die in der Mitte fast $100 \mathrm{~m}$ und am Nordrande längs des Sava-Flusses 10 bis $20 \mathrm{~m}$ tief ist. Die ganze Einsenkung ist mit karbonatreichem Schotter und Sand ausgefüllt. Die unteren, pleistozänen Schichten sind teilweise konglomeriert. Die obersten, holozänen Schotterschichten sind mit einer verschieden dicken Humusdecke versehen, die teilweise bewaldet und teilweise landwirtschaftlich bearbeitet ist, auf einem grossen Teil aber bebant und mit einer wasserundurchlässigen Asphaltdecke bedeckt ist. Die Länge des Ljubljansko polje beträgt etwa 15 und die Breite bis etwa 5 km; es zieht sich in der Richtung WNW-OSO (Breznik 1969, Zlebnik 1971).

Das Gebiet ist als Zentrum Sloveniens besonders in den letzten Dezenien grossen Veränderungen unterworfen worden. Zur natürlichen, allmählichen Einsenkung des Flussbettes in die stellenweise schon undurchlässige, permokarbonische Unterlage (Verkleinerung der Infiltrationsflächen) gesellen sich der Ausbau der Industrie flussaufwärts von Ljubljana und die wachsende Stadtpopulationen (starke anorganische und organische Verunreinigung), die Erweiterung der Stadtfläche (verkleinerte Fläch’en für die Infiltration des Regenwassers in das Grundwasser), der Ausbau der Reinigungsanlagen in allerletzter Zeit (eine sekundäre Verbesserung des Sava-Wassers), der erhöhte Grundwasserverbrauch (Erniedrigung des Grundwasser-Spiegels). Überdies verursachen die Wasserkraftwerke oberhalb Ljubljana kurzdauernde (manchmal tägliche) aber starke Verănderungen des Wasserstandes und der Wasserqualität.

Das Ljubljansko polje ist von fast allen Seiten von niedrigen, aus Schiefer- bzw. Sandsteinen bestehenden Bergen umgeben. Doch ein enger und seichter Schottergürtel zieht sich längs des ganzen Sava-Tales in Slovenien, der auch mehrere kleinere Karst-Massive berührt oder sogar überquert. Es existiert also wenigstens ein indirekter Kontakt des Grundwassers mit den unterirdischen Karstgewässern. Obgleich die Grundgewässer des Ljubljansko 


$$
3
$$


polje selbst vom Massiv des Dinarischen Karstes etwa lo km entfernts sind, könnte diese Bresche an zwei Stellen (im W und SO) überbrückt sein. Die verkarstungsfähigen Konglomeratschichten sind an diesen Stellen nähmlich den wenigstens schwach verkarsteten, inselartigen Kalkstein- bzw. Dolomitmassiven genähert oder mit ihnen sogar unterirdisch verbunden.

Unsere Untersuchungen - als erste intensivere Grundwasseruntersuchungen in Slovenien - sind schon im Jahre 1955 begonnen worden, doch waren sie damals nur faunistisch orientiert. Später haben wir intensiver die Dynamik der Tierpopulationen im ergiebigen Norton-Brunnen am Dovjez studiert, sowie etwas genauer die Verbreitung der Tiere in verschiedenen Teilen und Habitaten im Rahmen der Ebene. Wir haben die verschiedensten Sammelmethoden für die unterirdische Tiere angewandt (Filtrieren des Wassers aus Nortonbrunnen und Bohrungen, Karaman-Chappuis-Methode, BouRouch-Sonde, Cvetkov-Netz usw.). Den chemischen Untersuchungen haben wir dagegen vielleicht zu wenig Aufmerksamkeit gewidmet.

Mit der BR-Sonde haben wir das Grundwasser an jeder Stelle von einer Tiefe von $50 \pm 10 \mathrm{~cm}$ (im Flussbett) bzw. $20 \pm 10 \mathrm{~cm}$ (am Ufer) sowie von etwa $80 \pm 10 \mathrm{~cm}$ (in beiden Fällen) gepumpt (Abb. 2). Das Grobe Schotter ermöglicht tiefere Einsenkung der Röhre nicht. Die Menge des gefilterten Wassers wurde immer ausgemessen, das Verhältniss Wassermenge/Tierzahl kann uns aber keinen absoluten Wert der Besiedlungsdichte zeigen.

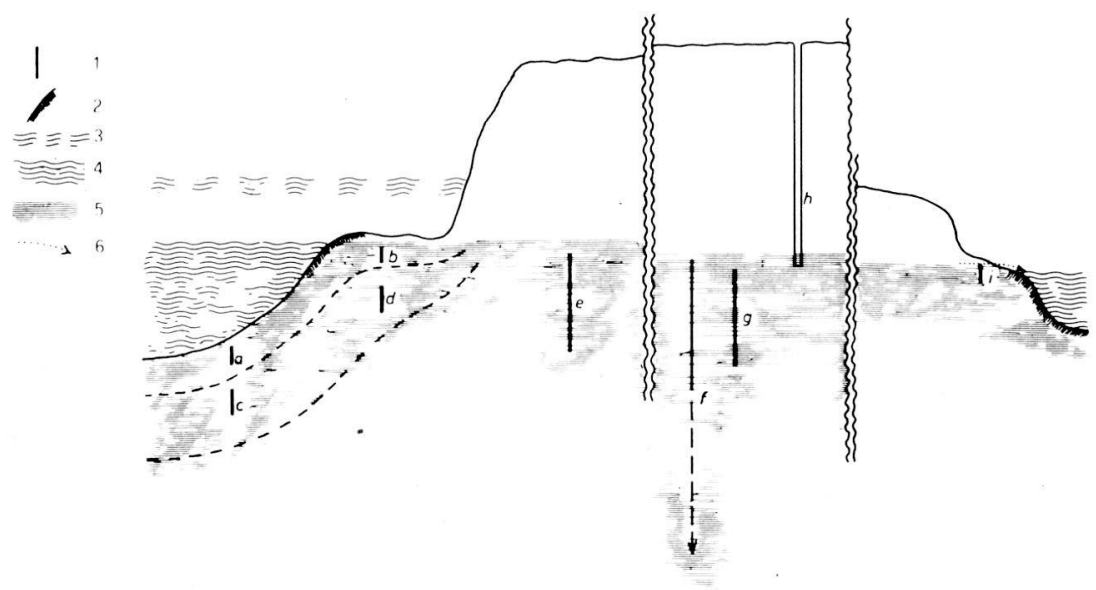

Abb. 2. Skizze der Sonden; 1 - Sonde (a-d - Bou-Rouch- und Karaman-Chappuis-Sonden im Bettboden und im Ufer, e - Nortonbrunnen in der Uferterrasse, $\mathrm{f}-\mathrm{g}$ - Tiefbohrungen und Nortonbrunnen im Inneren der Ebene, $h$ - Schachtbrunnen, i - BR-Sonde an der Ausflussquelle), 2 - wasserundurchlässige Uferzone, $3^{*}-4$ - Flusswasser, während des hohen und des niedrigen Wasserstandes (links Sava, rechts Ljubljanica), 5 - Grundwasser, 6 - Ausflussquelle. 


\section{HYDROGRAPHISCHE VERHÄLTNISSE}

Das Grundwasserbassin des Ljubljansko polje füllt sich vorwiegend durch die Schotter aus dem Flussbett in der NW-Ecke der Ebene. Kleinere Wassermengen gelangen hieher von einigen anderen Stellen längs des Sava-Flusses sowie vom Süden. Der Gesammtdurchfluss des Grundwassers wurde im ausgesprochen trockenem Herbst 1967 mit 1400 1/sec berechnet. Das Wasser erneuert sich mit Regen nur etwa mit $15 \%$. Der Grundwasserspiegel ist etwa gleich dem Spiegel des Sava-Flusses, dies Stromrichtung ist der des Sava-Flusses parallel. Die stromgeschwindigkeit des Grundwassers beträgt nur $10 \mathrm{~m} / \mathrm{Tag}$. Bis zu 75\% des Grundwassers wird in der Trockenperiode vom Wasserwerk der Stadt Ljubljana sowie von anderen Verbrauchern in der Mitte der Ebene ausgepumpt, der Rest des Grundwassers trit aber in mehreren Quellen am O-Ende der Ebene zutage (Breznik 1969).

Das Boden des Flussbettes ist vorwiegend aus Schotter bestehend, an seichten Uferstellen und in kleineren Einbuchtungen lagert sich Sand bzw. Detritus ab. Unsere Grundwasseruntersuchungen zeigen, dass das Wasser aus dem Sava-Flussbett an sanft geneigten Uferstellen sowie an seichten Stellen des Bett-Bodens jetzt nicht in die Schotterablagerungen eindringen kann. Ständige vom Wasserkraftwerk verursachte Wasserspiegeloszillationen hatten nähmilich eine Verdichtung der Lücken mit organischem Material (Sphaerotilus-Matten, Detritus) zur Folge. Als Infiltrationszone kommt also nur der tiefere Flussbettboden in Betracht, wo der Schotter ständig in Bewegung ist un wo er sich dadurch ständig reinigt. Weil das Flussbett stellenweise ziemlich stark geneigt ist, bildet das Grundwasser auch kleinere, vom Flusswasser unabhängige Strahlen, die, nur meterweit vom Flussrand entfernt, einen höheren oder niedrigeren Wasserspiegel als das Fluss an der nächsten Stelle, haben können. Die erwähnte verdichtete Isolationsschicht ist äusserst dünn und grenzt landwärts manchmal an sehr grosslückigen Schotter.

Die Schotterablagerungen des Ljubljansko polje, durch welche der Hauptstrom des Grundwassers fliesst, sind zwar mit Sand gemischt, scheinbar aber füllt der Sand auf fast keiner uns zugänglichen Stelle die Lücken vollkommen aus, so dass man das Habitat als eine Kombination des Stygopsephals und Stygopsammals bezeichnen könnte.

Die Quellen am Ostende des Ljubljansko polje springen teilweise aus leicht verkarsteten Konglomeratschichten, die aber wieder mit Schotter bedeckt sein können.

\section{EIGENSCHAFTEN DES WASSERS}

Das Gerbigsfluss Sava hat grosse Stromgeschwindigkeiten. Obgleich das Sava-Wasser längs des Polje mit schwankenden, aber mehrmals sehr hohen Mengen verschiedener Pollutanten verunreinigt ist, sind hier die $\mathrm{O}_{2}$ Verhältnisse sehr günstig. Bei verhältnissmässig niedrigen Temperaturen (etwa $5-17^{\circ} \mathrm{C}$ ) schwankt das $\mathrm{O}_{2}$-Deficit von 0 bis ausnahmsweise $30 \%$.

Über die Verteilung bzw. Verbrauch des Sauerstoffes im Grundwasser 
belehren uns sehr gut die Verhältnisse, die im Sommer (August 1978) während der Übersättigung des Sava-Wassers festgestellt wurden. Nur an seltenen Stellen im Flussbett sowie am Ufer erreicht bei etwa $50 \mathrm{~cm}$ Tiefe (im Substrat) die Sättigung 80-100\%, wahrscheinlich handelt sich hier um die Stellen, wo das Flusswasser intensiver in die Unterlage infiltriert. Sonst erträgt die $\mathrm{O}_{2}$ Sättigung des Grundwassers so unter und neben des Flussbettes, wie auch im Inneren der Ebene etwa 40-60\%. Eine ausgesprochen Sauerstoffarme Grundwasser-Schicht (vergleiche Husmann 1972) um das Flussbett wurde hier also nicht festgestellt. Auch die Verhältnisse in fliessenden Wasserstrahlen neben des Flusses scheinen keine Abweichungen davon zu vorstellen. In der Ausflussquelle im Osten des Feldes ist die Sauerstoff-Sättigung dagegen auf $75 \%$ erhöht.

Organische Detritus-Teilchen sind im Interstiziellwasser um das Flussbett ziemlich unregelmässig verteilt. Die Masse des Detritus kann bis zu metertief im Grundwasser von der Gesammtmasse der Tiere viel grösser aber auch etwas kleiner sein. In den Proben aus der Nortonbrunnen so längs des Flusses, wie auch im Inneren der Ebene haben wir dagegen kein Detritus mehr bekommen.

Die Menge der organishen Stoffe im Flusswasser ist sehr grossen Schwankungen unterworden. Der $\mathrm{KMnO}_{4}$-Verbrauch erreicht 2-60 mg/1, $\mathrm{BSB}_{5} 2-10 \mathrm{mg} / 1$. Bis zur Pumpstellen des Wasserwerkes erniedrigen und stabilisieren sich diese Werte auf etwa $2-5 \mathrm{mg} / 1 \mathrm{KMnO}_{4}$-Verbrauch und $\mathrm{BSB}_{5}$ von $0,3-0,8 \mathrm{mg} / 1$.

Die $\mathrm{NO}_{3}$-Menge scheint sich aber gegen die Pumpstelle (im Vergleich zum Flusswasser) zu vergrössern. Das ist im Einklang mit den Verhältnissen in den unterirdischen (also pflanzenlosen) Teilen der Sinkflüsse im Karste (Sket and Velkovrh 1981), stimmt aber nicht mit den Resultaten anderer Grundwasserforscher (Husmann 1972, 1975) zu, die vielleicht an stagnierenden oder flusswärts fliessenden Grundgewässern gearbeitet haben. Den Einfluss der Feld-Düngung auf den $\mathrm{NO}_{3}$-Gehalt des Grundwassers in Ljubljansko polje kann man selbtverständlich auch nicht ausschlüssen.

Die Temperatur-Amplitude des Sava-Wassers erreich hier etwa $15^{\circ}$ (kontinuierte Messungen nicht ausgeführt!) um einen Mittelwert von etwa $10^{\circ} \mathrm{C}$. Derselben jährlichen Temperaturschwankungen sind auch die Grundgewässer um das Flussbett (Nortonbrunnen in der Terrasse eingeschlossen) unterworfen. Auch die täglichen Schwankungen, die im Haurtstrom im Sommer etwa $6^{\circ}$ erreichen, sind in dem Bett und Seichtufer-Grundwasser noch ebenso hoch, nicht aber in den erwähnten Nortonbrunnen. Das Grundwasser in seichten Schotterbänken hat an der Oberfläche während der sonnigen Tage sogar um ein Paar Grad höhere Temperaturschwankungen. Das Grundwasser im Inneren des Feldes hat eine jährliche Amplitude von etwa $2^{\circ}$, um das Mittelwert von $11^{\circ} \mathrm{C}$.

\section{VERTEILUNG DER FAUNA}

Das aus grobem Schotter bestehende Stromgrund ist nur mit verschiedenen 
Algen (Cyanophyta, Bacillariophyta, Chlorophyta; stellenweise Sphaerotilus bis $20 \%$ der Bodenfläche) bewachsen. Von Tieren sind am zahlreichsten die Chironomidae-L. vertreten, weiter Gammarus fossarum Koch, Oligochaeta (Nais sp., Pristina spp. und Stylodrilus heringianus Claparede) und Leuctra spp. (Matoniĉkin und all. 1975, eigene Untersuchungen).

Selbstverständlich leben in dezimetertiefen Schichten des Schotterbodens regelmässig noch benthische Tiere, so vor allem Naididae, weiter

Tab. 1. Verteilung der Tiere in verschiedenen Habitaten (a-d, f-i - Bezeichnung wie in der Abb. 2; e - Nortonbrunnen am Dovjeẑ); in senkrechten Kolonen bedeutet die erste Nummer die Regelmässigkeit des Auftretens (1 - in vereinzelten Proben, $2- \pm$ regelmässig auftretend), die zweite die Zahl der Exemplare pro Probe (1 - vereinzelt, 2 - je bis 10 Exemplare, 3 - zahlreich bis massenhaft) $\left({ }^{+}\right.$nicht dieselbe Formen wie unter a-d).

\begin{tabular}{|c|c|c|c|c|c|c|c|c|c|}
\hline & a & b & c & d & e & f & g & h & $\mathbf{i}$ \\
\hline Nematodes gg.spp. & $2-1$ & $2-3$ & $2-2$ & $1-1$ & 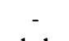 & - & - & - & -1 \\
\hline $\begin{array}{l}\text { Hadziella deminuta Bole } \\
\text { Hauffenia cf. michleri Kuŝcer }\end{array}$ & - & - & - & - & $1-1$ & - & - & - & - \\
\hline $\begin{array}{l}\text { (c,e,h) u. H.cf.erythropomatia } \\
\text { (Hauffen) (i) }\end{array}$ & - & - & $1-1$ & - & $1-1$ & - & - & -+ & -1 \\
\hline $\begin{array}{l}\text { Belgrandiella kuesteri Boeters } \\
\text { u. B.fontinalis (Schmidt) }\end{array}$ & - & $1-1$ & - & $1-2$ & - & - & - & - & -3 \\
\hline Bythinella schmidti (Küster) & $1-1$ & $2-2$ & $2-2$ & $2-2$ & - & - & - & - & -1 \\
\hline Naididae gg.spp. & $2-3$ & $2-2$ & $2-3$ & $1-1$ & - & - & - & - & - \\
\hline Oligochaeta (ex.Naididae) & $2-3$ & $2-3$ & $2-3$ & $2-3$ & $2-1$ & $2-1$ & $\therefore$ & $1-2$ & - \\
\hline Cyclopoida gg.spp. & $1-2$ & $2-3$ & $2-3$ & $2-3$ & $2-3$ & $?$ & $1-1$ & $1-2$ & - \\
\hline Harpacticoida gg.spp. & - & - & $1-1$ & - & $1-1$ & ? & - & - & -1 \\
\hline Ostracoda gg.spp. & - & $1-3$ & $1-1$ & $1-3$ & $2-1$ & ? & - & $1-1$ & - \\
\hline Bathynellacea gg.spp. & - & - & - & - & $1-1$ & $?$ & $1-1$ & - & - \\
\hline Proasellus deminutus (Sket) & - & - & $1-3$ & $2-3$ & $2-3$ & - & $1-1$ & - & - \\
\hline $\begin{array}{l}\text { P.vulgaris (Sket) (nur an } \\
\text { lenitischen Stellen) }\end{array}$ & - & - & $1-3$ & $1-3$ & - & - & - & - & -1 \\
\hline P.pavani orientalis (Sket) & - & - & - & - & $1-1$ & $1-1$ & - & $1-1$ & - \\
\hline Niphargus s.ljubljanensis Vodnik & - & $2-2$ & $2-1$ & $2-2$ & $1-1$ & - & - & $1-3$ & - \\
\hline N.stygius valvasori S.Karaman & - & - & $\therefore$ & - & - & - & - & $1-1$ & -2 \\
\hline N.longidactylus Ruffo & $1-2$ & $1-1$ & $2-3$ & $2-3$ & $2-3$ & $2-3$ & $1-1$ & $1-3$ & -2 \\
\hline N.pectinicauda Sket & $1-1$ & $1-2$ & $2-2$ & $2-3$ & $2-2$ & $1-1$ & $1-1$ & - & - \\
\hline N.pupetta (Sket) & - & - & - & - & $1-1$ & - & $1-1$ & $1-2$ & -1 \\
\hline N.serbicus S.Karaman & - & - & - & - & - & $1-1$ & $1-1$ & $1-1$ & -1 \\
\hline N.jovanovici multipennatus Sket & - & - & - & - & $2-2$ & $2-1$ & $2-1$ & $1-1$ & -1 \\
\hline Bogidiella spp. & - & - & - & - & $2-2$ & ? & $1-1$ & $1-1$ & - \\
\hline Chironomidae-1. & $2-2$ & $1-2$ & $2-1$ & $1-1$ & - & - & - & $1-2^{+}$ & - \\
\hline Tipulidae-1. & $1-1$ & $1-1$ & $1-1$ & - & - & - & - & $1-1^{+}$ & - \\
\hline Leuctra-1. & $1-1$ & - & $1-1$ & - & - & - & - & - & - \\
\hline $\begin{array}{l}\text { Stygoxene Formen (Pisidium, } \\
\text { Erpobdella, Lymnaea, Helmidae) }\end{array}$ & - & $2-1$ & - & - & - & - & - & $2-2^{+}$ & - \\
\hline Menge des Wassers pro Probe in $l$ & 30 & $\begin{array}{c}50 \\
\text { bis } \\
200\end{array}$ & 30 & $\begin{array}{l}40 \\
\text { bis } \\
80\end{array}$ & $\mathrm{~lm}^{3}$ & $\begin{array}{c}1000 \\
\mathrm{~m}^{3}\end{array}$ & & & \\
\hline $\mathrm{O}_{2}$-Sättigung in $\%$ (Sommer) & $\begin{array}{c}60 \\
\text { bis } \\
100\end{array}$ & $\begin{array}{l}40 \\
\text { bis } \\
80\end{array}$ & $\begin{array}{l}30 \\
\text { bis } \\
40\end{array}$ & $\begin{array}{c}30 \\
\text { bis } \\
50\end{array}$ & $\mathrm{x}$ & 50 & $\mathrm{x}$ & $\mathrm{x}$ & 75 \\
\hline
\end{tabular}


Chironomidae-L., Tipulidae-L., Leuctra-L.; die Insecten-Larven kommen aber nie in einer bedeutenderen Zahl vor. Copepoden sind hier selten, $\mathrm{Ni}$ phargen kommen nur stellenweise vereinzelt vor, andere unterirdische Tiere sind noch abwesend. Gegen metertief im Flussboden veränderen sich stellenweise die Verhältnisse zugunsten der stygobionten Tiere. Auch Cyclopoida kommen da schon massenhaft vor, Niphargus longidactylus bildet ziemlich dichte, $N$. pectinicauda spärlichere populationen, ebenso $N$. longicaudatus ljubljanensis; Proasellus deminutus ist ziemlich regelmässig vorhanden. Auch vereinzelte Insektenlarven leben noch hier. An den Stellen, wo diese ausgeprochen selten und Naididae sp̈ärlicher vertreten sind, kommt Proasellus schon massenhaft vor.

Auch im Grundwasser der seichten Ufer kommen in der Nähe des Wasserspiegels regelmässig benthische Tiere vor, obgleich dieses Wasser während des niedrigen Wasserstandes nicht sehr direkt mit dem Flusswasser verbunden ist. Scheinbar sind dies die Tiere, die während des rückziehens des Hochwassers in die Schotterbänke eindringen und da wegen der guten Ernährungverhältnisse überleben können. So findet man hier sogar die Arten, die im Stromm nicht aus dem Benthos ins Grundwaser aktiv eindringen, wie Hydra sp., Lymnaea sp., Sadleriana sp., Erpobdella sp., Coleoptera-L.(Dytiscidae, Helmidae). Oligochaeta (meistens auch Naididae) sind regelmässig zahreich vertreten, ebenso Cyclopoida und Nematoda. Da leben noch Niphargus longidactylus, N. pectinicauda und N.l.ljubljanensis, ihre Besiedlung kann sogar ziemlich dicht sein an solchen Stellen, wo die Oberflächen-Tiere fast verschwinden. Nur an solchen Stellen, wo kleine Grundwasserstrahlen aus dem Ufer ausfliessen, findet man bei der Oberfläche auch Proasellus vulgaris.

In Metertiefe ist die Zusammensetzung der Fauna fast jenen Faunen gleich, die man an einigen Stellen unter dem Flussstrom in der gleichen Tiefe findet und die als insektenarm bezeichnet wurden. Scheinbar vertritt an den Stellen mit bemerkbarem Wasserstrom Proasellus vulgaris den $P$. deminutus des stehenden Wassers. Die Naididae sind hier kaum noch vorhanden, ebenso die Insektenlarven.

An der höheren Uferterrasse waren im Gebiet der Hauptinfiltrationszonen drei Nortonbrunnen vorhanden, die nur je etwa $5 \mathrm{~m}$ vom Wasserrand des Flusses entfernt waren. Während zwei Brunnen beim Pumpen nur wenige Tiere lieferten, fanden wir in den Proben aus dem dritten, am Sava-Ufer bei Dovjeẑ, eine Fauna, die nicht nur individuen- sondern auch sehr artenreich war.

Die Nortonröhren reichen nur etwa metertief in das Grundwasser. Obgleich wir keine exacten Daten über die hydrographischen Verhältnisse haben, zeigen uns die Temperaturverhältnisse, sowie die Faunenzusammensetzung, dass der Einfluss des Flusses hier wieder ein wenig milder ist als bei den obenerwähnten, durch BR-Sonden erreichten Grundwasserstellen. Über den Kontakt mit dem Flusswasser sprechen hier die Temperaturverhältnisse; trotz einer etwa $3 \mathrm{~m}$ dicken Schotterschicht, die diese Stelle bedeckt, ist die Wassertemperatur einer jährlichen Schwankung von $14^{\circ}\left(4^{\circ}\right.$ bis $18^{\circ} \mathrm{C}$ ) unterworfen. Auch das - zwar ausserordentlich seltene - auftreten der ausgesprochen stygonen Cladocera in den Proben spricht für das periodi- 
sche Eindringen des wenig filtrierten Flusswassers in diese Stelle. Trotzdem findet man hier keine Chironomiden-Larven mehr.

Wegen des stetigen Pumpens musste sich in unmittelbarer Umgebung des Nortonbrunnens am Dovjê̂ ein geräumiges System sandloser Lücken in der Schotterschichten ausbilden. Man findet in den Proben auch keine DetritusTeilchen. Aus diesem Brunnen konnte man (1959-1965) im Durchschnitt je etwa 30-300 Exemplare auf 500-1000 l Wasser bekommen. Insgesammt wurden über 30 Tierarten gefunden (Taf. 2), beim jeden Pumpen je etwa 10 (bzw. 6-15) Arten. Mit durchschnittlich etwa 50\% der Exemplare je Probe hat im Jahre 1959 Proasellus deminutus überwogen, ihm folgen die Cyclopoida. Im Jahre 1964 haben beide Gruppen die Priorität ausgetauscht (Abb. 3). Niphargus (mit wenigstens 8 Arten) steht in beiden Fällen der zweitmächtigsten Gruppe wenig nach. In wenigen Exemplaren, aber sehr regelmässig kommen 2 Bogidiella spp. und Oligochaeta vor. Die zuerst in vereinzelten Exemplaren vorkommende Harpacticoida, Ostracoda, Bathynellacea, sind gegen Mitte 1964 fast ausgeblieben. Andere Formen treten mehr vereinzelt und unregelmässig vor (so nur je einmal Alona sp. und Chydorus sp.).

In anderen Nortonbrunnen längs des Sava-Ufers wurden nur vereinzelte

Tab. 2. Struktur der Fauna im Nortonbrunnen am Dovjeẑ (Sava-Ufer N von Ljubljana). Die erste Nummer bezeichnet die Regelmässigkeit des Auftretens ( 1 - bis 3 Proben, 2 - bis $50 \%$ der Proben, 3 - regelmässig oder konstant), die zweite die Häuffigkeit in einzelner Probe (1 - 1-2 Exemplare, 2 - 1-10 Exemplare, 3 - zahlreich). Es wurden etwa 10001 Wasser pro Probe gefiltert.

TURBELLARIA
Tricladida g.sp.
Turbellaria g.sp.A
Turbellaria g.sp.B
NEMATODES
Nematodes gg.spp.
GASTROPODA
Hadziella deminuta Bole
Hauffenia cf. michleri Kuŝcer
Mervicia eximia Bole
OLIGOCHAETA
Rhynchelmis sp.
Trichodrilus pragensis (Vejdovsky)
Oligochaeta gg.spp.
CYCLOPOIDA
Acanthoc. venustus (Norm. et Scott)
A. kieferi (Chappuis)
A.hispanicus Kiefer
Diac.languidoides (Lilljeb.)
Megac. viridis (Jurine)
HARPACTICOIDA
Nitocrella slovenica Petk.
Nitocrella hirta Chappuis

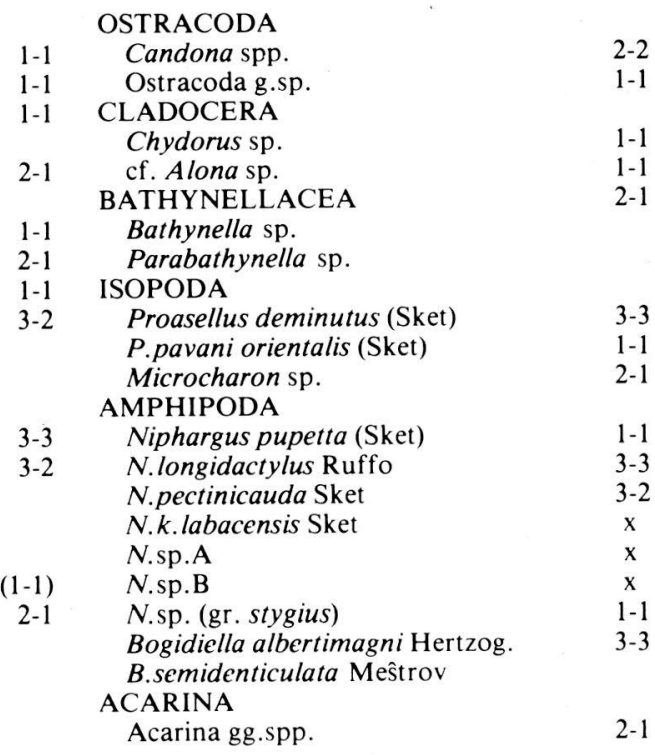


Exemplare der Niphargus-, Proasellus-, Bogidiella- und Cyclopoida-Arten ausgepumpt.

Im Inneren des Ljubljansko polje haben wir das Wassers, das aus den frischen, erst gereinigten, für die neue Wasserwerkstation gemachten Bohrungen gepumpt wurde, filtriert. Diese Bohrungen liefern uns das Wasser aus allen Schichten, vom Wasserspiegel (etwa $10 \mathrm{~m}$ unter der Feld-Oberfläche) bis zur Tiefe von $50 \mathrm{~m}$, gemischt. Die Temperatur beträgt $11 \pm 1^{\circ} \mathrm{C}$. Von der nächsten Stelle des Sava-Ufers sind diese Bohrungen etwa $2,5 \mathrm{~km}$ entfernt; das bedeutet, dass das hier gepumpte Wasser wenigstens (!) 100 Tage durch die filtrierenden Schotterschichten fliesst. Nach allen Eigenschaften zu urteilen (chemischen, bakteriologischen, organoleptischen) ist dies ein ausserordentlich gutes Trinkwasser. Die unten angeführten Daten über die Fauna gelten für das Versuchpumpen, beim welchen wir aus 4 Bohrungen insgesammt 5.000.000 1 Wasser filtriert haben. Zwei Jahre nach dem Beginn des regelmässigen Gebrauchs des Pumpwerkes konnte man in 380.0001 Wasser kein Tier finden. In der erwähnten Wassermenge fanden wir nur etwa 720 Exemplare des Niphargus longidactylus, je 30 Exemplare des N.jovanovici multipennatus und der Oligochaeta, je 10 bis 1 Ex. von Niphargus pectinicauda, Niphargus sp. indet., N.serbicus und Proasellus pavani orientalis. Die Copepoda könnten in einer kleiner Zahl vorhanden sein (mit der gebrauchten Methode nicht feststellbar), jedenfalls aber nicht zahlreich. Bezeichnend ist das vollkommene Abwesenheit des Proasellus deminutus. Erwähnenswert ist das allmähliche Verkleinern der Tierzahl während des Pumpens.

Auch die Fauna in (zwar schon sehr seltenen) Nortonbrunnen im Inneren der Ebene, die uns nur das Grundwasser der obersten Schichten liefern, ist äusserst individuen- und artenarm. Ihre Zusammensetzung ist zwar jener der Tiefbohrungen ähnlich, nur Niphargus longidactylus hier nur ausnahmsweise, Proasellus deminutus aber ziemlich regelmässig auftritt.

1959
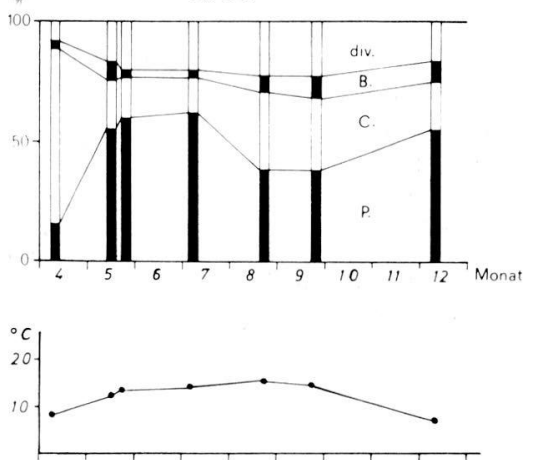

$1964 / 65$
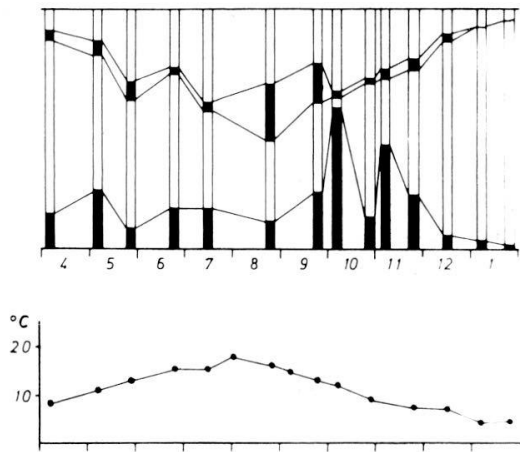

Abb. 3. Struktur der Fauna im Nortonbrunnen am Dovjê̌ während der Jahre 1959 und 1964; unten die Kurven der Wassertemperatur; P - Proasellus deminutus, C - Cyclopoida, B - Bogidiella spp., div. - andere Tierarten. 
Ein besonderes, fremdartiges Habitat in diesem Gebiete stellen die ausgegrabenen Schachtbrunnen vor. Es handelt sich hier nähmlich um grössere, mit Wasser ausgefüllte Räume, die für ausgesprochen tygmotaktische Tiere ungünstig, für die Tiere mit grösseren Körperausmassen aber äusserst günstig sein können. Die Temperaturschwankungen des Wassers können gegenüber dem "normalen" Grundwasser der Ebene nur leicht vergrössert sein. Eine äusserst günstige Erscheinung, welche die Entwicklung dichteren Tierpopulationen ermöglicht, ist das stettige Vorhandensein einer futterreichen Detritusschicht am Boden der Schachtbrunnen. Solche Brunnen sind im Gebiet fast nicht mehr vorhanden, denn die alten sind wegen des erniedrigten Grundwasserstandes trocken geworden. In den Brunnen waren sonst regelmässig Oligochaeta, Cyclopoida, manchmal Diptera-Larven vorhanden, ausserdem gewöhnlich ziemlich dichte Population einer Niphargus-Art. In einem Fall war dies der winzige N.longidactylus, in einigen anderen der grosse Niphargus "longicaudatus" ljubljanensis. Man muss bemerken, dass ausserhalb des oberen Teiles des Sava-Systems solche Brunnen sehr oft und dicht Proasellus slavus sspp. besiedeln. Von den Quellen am Ost-Ende des Ljubljansko polje haben wir eine kleine Rheocrene untersucht, die aus leicht verkarsteten Konglomeraten durch eine Schotteransammlung fliesst. Während in der Quelle selbst schon epigäische Tiere wie Gammarus fossarum Koch, Belgrandiella fontinalis und B.kuesteri massenhaft vorkommen, aber auch für die Kluftgewässern des Karstes charakteristische Niphargus stygius und Hauffenia erythropomatia (Hauffen) vorhanden sind, lieferte uns eine unmittelbar oberhalb des Ausflusses eingehammerte BR-Sonde auch typische Bewohner des Interstizialwassers. Beide Belgrandiella-Arten, die im Ufergrundwasser des SavaFlusses äusserst selten vorkommen, sind hier ziemlich dicht besiedelt. Niphargus longidactylus, N.pupetta, N.serbicus, N.j.multipennatus kommen in wenigen Exemplaren vor, ebenso aber auch Proasellus vulgaris und Hauffenia cf. erythropomatia. Charakteristisch ist das Fehlen der Cyclopoida.

\section{ÖKOLOGISCHE UND ZOOGEOGRAPHISCHE CHARAKTERISIERUNG EINIGER FAUNENELEMENTE}

Bythinella schmidti tritt im behandelten Gebiete regelmässig im Grundwasser an solchen Stellen auf, wo auch die Insecten-Larven noch vorhanden sind. Sie ist also auf die Einflusszone des Flusswassers gebunden, obgleich sie im Benthos des Flusses nicht vorkommt. Alle Exemplare sind (die Augen ausgenommen) weitgehend depigmentiert. Sonst ist diese Schneckenart in Slovenien weit verbreitet (Sava-System, Karstgebiete eingeschlossen; Radoman 1976). Sie lebt in Quellen und Bächen, so auf kalkigen als auch auf SilikatUnterlage und wurde oft in Höhlengewässern und in ausgegrabenen Brunnen gefunden.

Belgrandiella fontinalis und B.kuesteri treten im Grundwasser längs des Flusses nur vereinzelt und im Inneren des Ljubljansko polje überhaupt nicht vor, massenhaft aber in den Ausflussquellen. Beide Arten sind in Slovenien (ausser des NO) weit verbreitet (Radoman 1975), der obengenannten Bythi- 
nella ökologisch ähnlich; auch sie zeigen eine ausgesprochene Neigung zum eindringen in die unterirdische fliessende Karstgewässer.

Hadziella deminuta findet man vereinzelt im Grundwasser des SavaSystemes in Slovenien. Dieselbe (oder sehr ähnliche) Form tritt auch in den kleinen Karstquellen desselben Gebietes auf. Diese Form ist von der Arten des Dinarischen Karstes morphologisch ziemlich weit entfernt.

Proasellus deminutus scheint eine ziemlich hoch spezialisierte interstiziellform zu sein. Sie wurde fast nie im futterreichem Potamostygon in Gesellschaft mit den epigäischen Tieren gefunden, überhaupt nie in den ausgegrabenen Brunnen oder in den Karstgewässern. Trotzdem bildet diese Form dichtere Populationen nur stellenweise, in der Nähe der Flüsse, wo sie auch grösseren Temperaturschwankungen unterworfen sein kann. Diesem anscheinenden Hapthabitat entsprechend hat P.deminutus eine ausgesprochene Reproductions-Periodizität. Die Zahl der $\odot$ mit Oostegiten ist im Mai, die Zahl der freien Jungen im Juli am höchsten. Vom September bis März findet man im Nortonbrunnen am Dovjeẑ nur vereinzelte, ausgewachsene, reproductionsunaktive Tiere (Sket 1965).

Proasellus vulgaris vulgaris (früher als Asellus deminutus vulgaris beschrieben) ist der obenerwähnten Form ohne Zweifel sehr nahe verwandt. Er wurde zuerst in den Höhlengewässern am Rande des Dinarischen Karstes und in inselartigen Karstmassiven gefunden, später aber auch in interstiziellen Gewässern der weiteren Umgebung des Ljubljansko polje, doch immer in fliessenden unterirdischen Wasserstrahlen (in "Schotterquellen"). Er scheint dem $P$.deminutus gleiche Reproductionsperiodizität zu haben.

Die ganze deminutus-Gruppe stellen sehr kleine (unter $5 \mathrm{~mm}$ lange) und ziemlich schlanke Tiere vor, deren Besonderheit ein sehr stark erweiterter Endit des I.Maxillen-Basale ist. Diese Erscheinung könnte der erweiterten Maxille des Niphargopsis analog sein und vielleicht dieselbe Rolle bei der Ernährung mit feinen Partikeln spielen. Alle morphologische Eigenschaften zeigen uns, sich diese Gruppe in interstiziellen Gewässern entwickelt und erst später teilweise in die Höhlenhabitate eingedrungen ist. Das bekannte Areal der Gruppe umfasst die nordwestlichsten Teile des Dinarischen Karstes und die Schotteransammlungen längs der grösseren Flüsse in NW-Jugoslavien und NO-Italien (Sket 1965, unveröffentl. Daten). Mit dieser Gruppe sind P.pavani Arcangeli und P.beroni Henry et Magniez aus Slovenien, NO-Italien und Korsika verwandt, die auch ökologisch wenig spezialisierte unterirdische Formen zu sein scheinen.

Niphargus longidactylus ist vielleicht die am weitesten verbreitete Tierform des Interstizielwassers so im Ljubljansko polje als auch in Slovenien überhaupt (Abb. 4). Er kommt ebenso in den Flussnahen wie auch in den flussfernen Grundgewässern vor, scheint aber das Potamostygon nur spärlich zu besiedeln. N.longidactylus scheint grössere Interstizien vorzuziehen und .bildet manchmal auch in ausgegrabenen Schachtbrunnen sehr reiche Populationen; trotzdem wurde diese Art in Jugoslavien nie in einem Karstwasser gefunden. N.longidactylus scheint in bevorzugtem Habitat äusserst konkurrenzfähig zu sein, seine Abbundanz ist oft sehr hoch.

N.longidactylus wurde bisher in NO-Italien (Verona) sowie in 
N-Slovenien, W-Kroatien und Bosnien gefunden, an einigen Stellen in nächster Nähe des Karstes, doch immer ausserhalb desselben. Andere Arten seiner Gruppe, (transitivus-Gruppe nach Sket 1971), die innerhalb der Gattung morphologisch ziemlich abweichend ist, leben im Gebiet des Dinarischen Karstes, auch nördlich davon und in Rumänien, selten auch in Höhlenseen.

Der derselben Gruppe angehörende aber grössere N.pectinicauda scheint für den Oberlauf des Sava-Flusses endemisch zu sein, wo er regelmässig, aber immer in einer weit kleineren Zahl den N.longidactylus begleitet. N.pupetta ist dagegen im Grundwasser zwischen NO-Italien und W-Rumänien weit verbreitet, wurde aber im Ljubljansko polje nur in insektenlosem Grundwasser gefunden, ausserdem nie in unterirdischen Karstgewässern des Dinarischen Karstes.

Niphargus jovanovici multipennatus scheint an das Interstizialwasser gebunden zu sein, zeigt aber hiefür keine Spezialisierung (Sket 1972). Er kommt in den Grundgewässern der feineren wie auch der grosskörnigeren Substraten, so im Potamostygon, wie auch in Eustygon vor. Nach unseren Erfahrun-

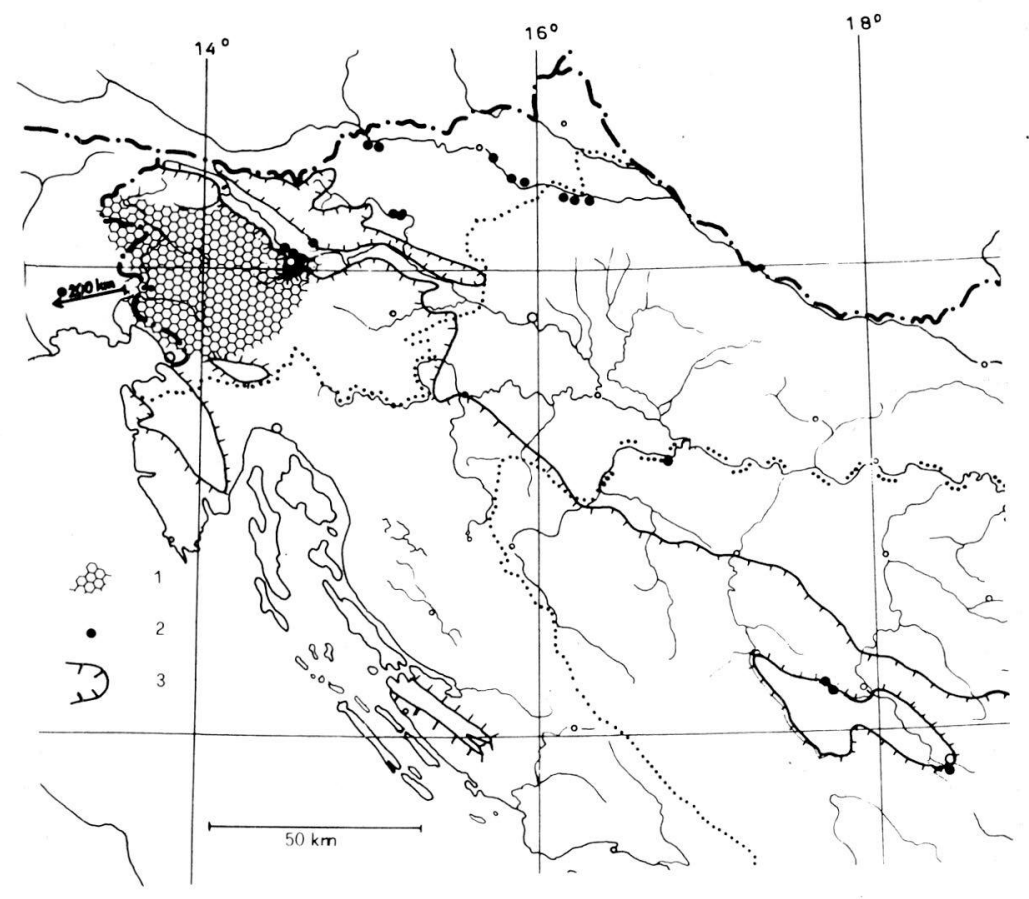

Abb. 4. Verbreitung des Niphargus stygius (Schioedte) und Niphargus longidactylus Ruffo; die letzterwähnte Art wurde auch bei Verona gefunden und ist wahrscheinlich in Friuli weiter verbreitet; 1 - N.stygius, 2 - N.longidactylus, 3 - Grenzen der grösseren Karstmassive (nur in Grenzen Jugoslaviens eingezeichnet). 
gen sind jedoch seine Populationen immer äusserst schütter, in keinem Habitat haben wir eine dichtere Besiedlung feststellen können. Nur ausnahmsweise wurde er in Schachtbrunnen gefunden, nie aber in Höhlengewässern.

Diese Form ist in den Schotterablagerungen längs der Sava wenigstens von NW-Slovenien bis Zagreb verbreitet, scheint aber auch in Bosnien vorzukommen.

Niphargus serbicus ist in faunareichem Grundwasser des Ljubljansko polje eine äusserst seltene Erscheinung. Es handelt sich wahrscheinlich um eine äusserst konkurrenzschwache Tierart, die nur an solchen Orten lebt, wo fast keine andere Tiere workommen. Im Potamostygon wurde sie nie gefunden. N.serbicus scheint grössere Interstizien zu bevorzugen und lebt auch in den Schachtbrunnen, nie aber in Höhlengewässern. Fast ausnahmslos wurden nur vereinzelte Exemplare gefunden.

Die Niphargus-jovanovici-Gruppe, zu welcher beide vorgenannten Formen gehören, ist in Europa weit verbreitet, alle Formen sind an das Interstiziallwasser gefunden. Nur zwei davon kommen ausnahmsweise auch in unterirdischen Gewässern des Karstrandes vor. Keine wurde bisher im Inneren des Dinarischen Karstes gefunden.

Niphargus "longicaudatus" ljubljanensis Vodnik (unveröffentlicht) ist die grösste unterirdische Tierart des Ljubljansko polje. Er kommt vor allem in Schachtbrunnen vor, wo er ziemlich dichte Populationen bilden kann. Man findet aber vereinzelte Tiere auch im Potamostygopsephal und auch im Eustygon, was seine Fähigkeit, sich durch die Interstizien auszubreiten, dokumentiert. Doch wurden an solchen Stellen immer nur junge Tiere gefunden. In den Brunnen, wo diese Form in reicheren Populationen gefunden wurde, variierte die Temperatur während des Jahres um einige Grade (in Brunnen in Savlje 9,5-12,9 ${ }^{\circ}$ ). Die Bruttragenden $q$ wurden nur in der Zeit vom Juli bis Dezember gefunden (Vodnik, unveröff.).

Diese Form wurde bisher mit Sicherheit nur in den obenerwähnten Habitaten längs eines kürzeren Sava-Abschnittes gefunden, doch ist ihr Vorkommen in den umgebenden Karstgewässern nicht ausgeschlossen (hierzu wäre eine Revision des Materiales nötig). Diese Niphargus-Form ist jenen sehr nahe verwandt, die Karaman (1952) als Unterarten des N.stygius aus NOSlovenien und W-Kroatien beschrieben hat. Sie leben in Schachtbrunnen sowie in unterirdischen Karstgewässern aller Typen. Allerdings gehören alle diese Taxa in die weitere Gruppe stygius-puteanus, die einige sehr euryöke Formen enthält. Ihr Areal bedeckt grosse verkarstete und unverkarstete Teile Europas. Niphargus stygius (Schioedte) wurde längs der Sava nur an zwei Stellen im Schotter gefunden, immer beim Ausfluss des Grundwassers, mit Proasellus vulgaris zusammen. N.stygius bzw.seine Forma valvasori lebt in Karstgewässern W-Sloveniens. bei den oben erwähnten Populationen konnten wir als einzigen Unterschied gegenüber valvasori nur eine kleinere Körperlänge feststellen.

Die Bogidiella spp., Microcharon sp. (cf. acherontis) und Bathynellacea wurden in Dinarischem Karste nur ausnahmsweise in Höhlengewässern gefunden, innerhalb des interstiziellen Lebensraumes zeigen sie aber keine zu grosse Spezialisierung. 


\section{SCHLÜSȘE}

Der Grundwasserkörper des Ljubljansko polje (NW-Jugoslavien) bekommt sein Wasser hauptsächlich aus dem Flussbett des Sava-Flusses, an einigen Stellen sind aber offensichtlich auch Verbindungen mit Karstgewässern vorhanden. Als Hauptinfiltrationszone kommt der Bettboden im Strom und nicht das mit organischen Abfällen verstopfte Ufer in Betracht. Die Hauptmasse des Grundwassers bewegt sich mit einer Geschwindigkeit von 10 $\mathrm{m} / \mathrm{Tag}$, doch bilden sich stellenweise kleine Wasserstrahlen mit ansehnlicheren Geschwindigkeiten.

In der unmittelbarer Nähe des Flusses sind die Jährlichen Temperaturschwankungen beträchtlich, in der Mitte des Ljubljansko polje erreichen sie kaum ein Paar Grad. Die $0_{2}$-Sättigung des Grundwassers erniedrigt sich schon etwa $1 \mathrm{~m}$ von gesättigtem Flusswasser entfernt, auf etwa 40-60\%.

Obgleich die Interstizien in Schotterschichten teilweise von feineren Sedimenten verstopft sind, sind hier ausgesprochen stygopsammale Tierformen nur mit vereinzelten Exemplaren und Arten vertreten. Bemerkbar ist auch die Armut an Nematoden un das fast vollkommene Abwesenheit der Acarina (vergleiche Danielopol 1976).

Bewohner des Fluss-Benthos (Chironomidae, Tipulidae, Leuctra spp., Baetis spp.) dringen in das untersuchte Grundwasser nur in wenigen Exemplaren ein (vergleiche Ruffo 1961, Danielopol 1976), nur die Naididae sind da zahlreicher vertreten. Trotzdem findet man im Grundwasser der nur periodisch überfluteten Schotterbänke auch dem Grundwasser vollkommen fremde Tiere, was eine indirekte Folge der Wasserstand-Oszillationen sein muss. Ziemlich regelmässig sind im flussnahen Grundwasser epigäische (creno- und troglophile) Tiere vorhanden, die im Fluss selbst überhaupt nicht gefunden wurden.

Als Faktoren, welche die Verteilung einzelner Arten innerhalb des untersuchten Grundwasserkörpers bedingen, kommt vor allem die Anwesenheit der Futteransammlungen und die damit bedingte Kompetition unter den Arten, weiter die Wasserströmung und einige schwieriger definierbare Faktoren in Betracht. Die Substratbeschaffenheit, Durchlüftung und andere Wassereigenschaften scheinen in diesem Falle keinen zu grossen Einfluss auf die Fauna zu haben.

Während mehrere Tierarten die futterreiche Nähe des Flusses ziemlich dicht besiedeln, trifft man im Inneren des Ljubljansko polje auf eine Armut an Arten- als auch Individuenzahlen. Dafür findet man aber hier einige Arten, die in der futter- (und konkurrenz-) reichen Zone nicht oder kaum vorhanden sind, ziemlich regelmässig. Die Wasserbewegung scheint die Ansiedlung einiger Formen (Cyclopoida, Proasellus deminutus) zu verhindern, andere Formen (P.vulgaris) sind dagegen an die Wasserströmung gebunden.

Einige Arten sind im Rahmen des Stygopsephals äusserst euryök ( $\mathrm{Ni}$ phargus longidactylus), andere dagegen sehr spezialisiert. Einige bilden sehr dichte Populationen und sind auch in energetisch ungünstigen Verhältnissen noch ziemlich dicht besiedelt, einige vermögen nur in günstigsten Bedingun- 
gen dichte Populationen zu bilden und einige scheinen überhaupt eine niedrige Abundanz zu haben.

Zoogeographisch betrachtet ist die behandelte Fauna aus recht verschiedenen Elementen zusammengesetzt. Es gibt hier die Tiere, die in Europa weit verbreitet sind und von Mitteleuropa bis zur Grenzen des Dinarischen Karstes(Bogidiella albertimagni) oder sogar ins Karst selbst (Trichodrilus pragensis, Acanthocyclops kieferi) eindringen. Auf die unterirdischen Gewässer des Oberlaufes des Sava-Flusses selbst scheinen Bogidiella semidenticulata, $\mathrm{Ni}$ phargus pectinicauda und Hadziella deminuta beschränkt zu sein. In allen Fällen handelt es sich um die Tiere, deren Haupthabitat die interstiziellen Gewässer sind un nur sporadisch in den unterirdischen Karstgewässern auftreten.

Niphargus stygius ist hier die enzige Tierform, die bestimmt aus der Karstgewässern stammt, und er ist im Grundwasser des Feldes auf ein ganz eigenartiges Habitat beschränkt. Es scheint, dass sich die ganze Proasellus deminutus-Gruppe am Kontakt des Karstes mit den Interstizielgewässern grosser Ebenen entwickelt hat, und dass einige Formen eher aus der Interstiziellgewässern in das Karst eingedrungen sind, als umgekehrt. Dagegen scheint (nach der Verbreitung der Gruppen beurteilend) für die Gattungen Hauffenia und Hadziella das Karst das Ursprungsgebiet zu sein. Eine so scharfe Abgrenzung der Höhlenfauna von der phreatischen in diesem Gebiet ist recht merkwürdig. Beide Faunen leben hier in einem grossen Reichtum in einem räumlich engem Kontakt. Es steht dem Gedanken nahe, dass gerade durch die Vielfältigkeit der beiden Faunen verursachte hohe Kompetition (und Spezialisierung) eine Mischung der Arten verhindert.

\section{LITTERATUR}

BARTHELEMY, C. 1968. Contribution a la connaissance des Leuctridae. Ann. Limnol. 4(27):175-198.

BOLE, J., 1967. Polẑi iz freatiĉnih voda Jugoslavije. Razprave SAZU, Cl. IV, 10(3):111-120.

BOU, C. 1968. Faune souterraine du sud-ouest du Massif Central, II.- Contribution a la connaissance de la faune des eaux souterraines de l'Albigeois. Ann.Speleol. 23(2):441-473.

BREZNIK, M. 1969. Podtalnica Ljubljanskega polja in moẑnosti njenega poveçanega izkoriŝcanja. Geologija (Ljubljana) 12:165-184, 2 Beilagen.

DANIELOPOL, D. 1976. The distribution of the fauna in the interstitial habitats of riverine sediments of Danube and Peisting. Int.J.Speleol. 8:23-51.

HUSMANN, S. 1966. Versuch einer ökologischer Gliederung des interstitiellen Grundwassers in Lebensbereiche eigener Prägung. Arch.Hydrobiol. 62(2):231-268.

HUSMANN, S. 1972. Das ökologische Gleichgewicht im Grundwasser sandig-kiesiger Ablagerungen usw., Tagungsber.Gesellschaft f.Ökol., Giessen 89-96.

HUSMANN, S. 1975. Versuche zur Erfassung der vertikalen Verteilung von Organismen und chemischen Zubstanzen im Grundwasser von Talauen und Terrassen; Methoden und erste Befunde. Int.J.Speleol. 6:271-302.

ILLIES, J., and L. BOTOSANEANU 1963. Problemes et methodes de la classification et de la zonation ecologique des eaux courantes, consideres surtout du point de vue faunistique. Mitt.Int.Ver.Limnol. 12:1-57.

KARAMAN, S. 1952. Podrod Stygoniphargus u Sloveniji i Hrvatskoj, Prirodoslovna istraz̃ivanja JAZU, Zagreb 25:5-38. 
LATTINGER-PENKO, R. 1976. Quelques donnees sur la population de Proasellus slavus ssp.n. Sket (Crustacea, Isopoda) dans l'hyporheique de la riviere Drave pres de Legrad. Int.J.Speleol. 8:107-115.

MATONIĈKIN, et al. 1975. Prilog valorizaciji voda ekosistema rijeke Save, Zagreb $96 \mathrm{pp}$.

MEŜTROV, M. 1960. Faunisticko-ekoloŝka i biocenoloŝka istraẑivanja podzemnih voda Savske nizine. Biol.Glasnik 13:73-109.

MESTROV, M. 1961. Über neue Bogidiella-Arten (Crustacea, Amphipoda) aus unterirdischen Gewässern Kroatiens und Sloweniens. Zool.Anz. 167(1/2):74-80.

MOTAS C. 1962. Procede des sondages phreatiques - division du domaine souterrain - classification ecologique des animaux souterrains - le psammon. Acta.Mus.Macedonici Sc.Nat. 8(7):135-173.

RADOMAN, P. 1975. Specijacija u okviru roda Belgrandiella i njemu srodnih rodova na Balkanskom poluostrvu. Glasnik Prir.Muz. (Beograd), Ser.B., 30:29-69.

RADOMAN, P. 1976. Speciation within the family Bythinellidae on the Balkans and Asia Minor. Z.f.zool.Systematik u.Evolutionsforschung 14(2):130-152.

RUFFO, S. 1961. Problemi relativi allo studio della fauna interstiziale iporreica. Boll.Zool. 28(2):273-319.

SKET, B. 1965. Subterrane Asellus-Arten Jugoslaviens (Crustacea, Isopoda). Acta Mus.Macedonici Sc.Nat. 10(1):1-26, 14 Beil.

SKET, B. 1971. Vier neue aberrante Niphargus-Arten (Amphipoda, Gammaridae) und einige Bemerkungen zur Taxonomie der Niphargus-ähnlichen Gruppen. Razprave SAZU, Cl.IV 16:1-25, 1 Beil.

SKET, B. 1972. Die Niphargus jovanovici-Gruppe (Amphipoda, Gammaridae) in Jugoslawien und NO-Italien, taxonomisch, zoogeographisch und phylogenetisch betrachtet, Razprave SAZU, Cl.IV 15:98-141, 2 Beil.

SKET B. et F. VELKOVRH, 1981. Postojna-Planina-Cave system as a model for the investigations of the polluted subterranean rivers, Naŝe jame 22 (in press).

STANFORD, J.A., and A.R. GAUFIN 1974. Hyporheic communities of two Montana rivers. Science 185:700-702.

VODNIK, F. (unveröffentlicht). Prispevek k poznavanju nifargov iz talne vode na Posavju pri Ljubljani, manuscr. 5 pp.

ŻLEBNIK, L. 1971. Pleistocen Kranjskega, Sorŝkega in Ljubljanskega polja. Geologija (Ljubljana) 14:5-51, 4 Beilagen. 